\title{
Inclusive hadron production in the CERN-LHC era
}

\author{
Rodolfo Sassot* and Pia Zurita ${ }^{\dagger}$ \\ Instituto de Física de Buenos Aires, CONICET, Departamento de Física, Facultad de Ciencias Exactas y Naturales, \\ Universidad de Buenos Aires, Ciudad Universitaria, Pabellón 1 (1428) Buenos Aires, Argentina \\ Marco Stratmann \\ Institut für Theoretische Physik, Universität Regensburg, 93040 Regensburg, Germany \\ and Institut für Theoretische Physik und Astrophysik, Universität Würzburg, 97074 Würzburg, Germany
}

(Received 4 August 2010; published 12 October 2010)

\begin{abstract}
We present a detailed phenomenological analysis of single-inclusive hadron production at the CERN-LHC in both proton-proton and proton-lead collisions. First data from the LHC experiments on charged hadron spectra are compared to next-to-leading order QCD expectations, and predictions are made for identified pion, kaon, and proton distributions differential in transverse momentum and rapidity for LHC energies from $900 \mathrm{GeV}$ to $14 \mathrm{TeV}$. The results are obtained with the latest sets of vacuum fragmentation functions based on global QCD analyses, and recently proposed medium modified fragmentation functions are used to model hadronization in proton-lead collisions assuming standard QCD factorization. Besides estimating theoretical ambiguities due to the choice of factorization and renormalization scales and parton densities, we carefully assess uncertainties due to our present knowledge of parton-to-hadron fragmentation functions with the Lagrange multiplier technique. It is outlined to what extent future LHC data will contribute to further our quantitative understanding of hadronization processes.
\end{abstract}

DOI: 10.1103/PhysRevD.82.074011

PACS numbers: 13.87.Fh, 12.38.Bx, 13.85.Ni

\section{INTRODUCTION AND MOTIVATION}

Single-inclusive hadron production is increasingly becoming a precise and versatile tool to quantitatively study various aspects of QCD, supplementing the insights already gained from fully inclusive observables. First and foremost, precise data obtained in electron-positron, lepton-nucleon, and hadron-hadron collisions at different center-of-mass system (c.m.s.) energies $\sqrt{S}$ have established collinear factorization [1] as the foundation for a perturbative QCD (pQCD) approach to hard scattering processes involving identified hadrons produced at large transverse momentum $p_{T}$.

Within the framework of collinear factorization, the presence of a hard scale $p_{T}$ allows one to compute with remarkable phenomenological success various kinematic distributions of the observed final-state hadron $H$ as a convolution of partonic short-distance scattering cross sections, calculable as a perturbative expansion in the strong coupling $\alpha_{s}$, and universal but nonperturbative functions capturing the long-distance physics as represented by parton distribution and fragmentation functions.

By now, information on these nonperturbative inputs is routinely extracted in "global QCD analyses" by consistently comparing data sets from many different experiments and processes with theoretical expectations at a given order in perturbation theory. In particular for parton density

\footnotetext{
*sassot@df.uba.ar

pia@df.uba.ar

marco@ribf.riken.jp
}

functions (PDFs), such type of analyses have reached a high level of sophistication in recent years [2-4]. Various methods have been put forward to arrive at faithful estimates of the remaining uncertainties in our understanding of the hadronic structure [4-6] and how they affect, e.g., the level at which we have control of standard model background processes at the CERN Large Hadron Collider (LHC).

Extractions of parton-to-hadron fragmentation functions (FFs) and their uncertainties are a much more recent achievement [7-9], mainly hampered by the considerably smaller amount of experimental results available. In general, reducing uncertainties of FFs is of utmost importance to further our understanding of the hadronization process, test the scale dependence of FFs as predicted in pQCD, and to delineate kinematic regions where factorized, colorindependent, collinear parton-to-hadron fragmentation provides a sensible approximation.

The limited knowledge of FFs has a serious impact on different studies, such as, for instance, global QCD analyses of data taken in collisions of longitudinally polarized nucleons and leptons aiming to address the fundamental question of how the spin of the nucleon is composed of the intrinsic spins and orbital angular momenta of quarks and gluons [10]. Since many of the data available so far involve identified hadrons in the final-state, either in semi-inclusive deep-inelastic lepton-nucleon or in proton-proton scattering processes, FFs are a crucial ingredient of theoretical analyses of the spin structure of the nucleon. Processes with identified hadrons are also increasingly used as decisive probes to gain insight about the properties and nature 
of cold and hot nuclear matter in heavy ion collisions both at the BNL Relativistic Heavy Ion Collider (RHIC) and soon also at the LHC. Again, precise knowledge of FFs and possible modifications induced by the nuclear medium are crucial ingredients that have received increasing attention recently [11].

In the following, we will assess in some detail how upcoming data from the LHC on high $p_{T}$ hadron production in an unprecedented kinematic regime will help to further our knowledge of FFs both in the vacuum and in a nuclear environment. To this end, we first need to discuss briefly some theoretical preliminaries such as the formalism for single-inclusive hadron production within pQCD factorization, the range of applicability of universal vacuum FFs, estimates and propagation of uncertainties, and a recent proposal for medium modified FFs.

\section{A. Framework for hadron production at the LHC}

Throughout this paper, we are interested in the singleinclusive invariant cross section for the production of a hadron $H$ with energy $E$ and momentum $\vec{p}$ in hadronhadron or hadron-nucleus collisions. Assuming pQCD factorization, the relevant theoretical expression for $p p$ collisions at the LHC schematically reads

$$
\begin{aligned}
E \frac{d^{3} \sigma^{H}}{d \vec{p}}= & \sum_{a, b, c} f_{a}\left(x_{a}, \mu_{f}\right) \otimes f_{b}\left(x_{b}, \mu_{f}\right) \otimes D_{c}^{H}\left(z_{c}, \mu_{f^{\prime}}\right) \\
& \otimes d \hat{\sigma}_{a b \rightarrow c X}\left(S, \alpha_{s}, x_{a}, x_{b}, z_{c}, \mu_{f}, \mu_{r}, \mu_{f^{\prime}}\right),
\end{aligned}
$$

where the sum is over all contributing partonic subprocesses $a b \rightarrow c X$ contained in the perturbatively calculable short-distance scattering cross sections $d \hat{\sigma}_{a b \rightarrow c X}$.

The scales $\mu_{f}$ and $\mu_{f^{\prime}}$ are introduced to factorize initial and final-state collinear singularities into the scale dependent PDFs and FFs, $f_{a, b}\left(x_{a, b}, \mu_{f}\right)$ and $D_{c}^{H}\left(z_{c}, \mu_{f^{\prime}}\right)$, respectively. $\mu_{r}$ denotes the energy scale at which $\alpha_{s}$ is being renormalized. The residual dependence of Eq. (1) on the arbitrary scales $\mu_{f, f^{\prime}, r}$ can be taken as a measure for the theoretical ambiguity due to the truncation of the perturbative series at a given fixed order in $\alpha_{s}$. To estimate its impact, we will follow the usual procedure and vary the scales within a factor of 2 around the default choice $p_{T}$. It turns out that scale variations are the dominant theoretical uncertainty for hadron production at the LHC.

$x_{a, b}$ are the fractions of longitudinal momentum of the colliding hadrons taken by the interacting partons $a$ and $b$. Similarly, $z \equiv z_{c}$ denotes the collinear momentum fraction of the fragmenting parton $c$ carried by the produced hadron $H$. Neither $x_{a, b}$ nor $z$ are measurable quantities, and any given data point characterized by the $p_{T}$ of the hadron $H$ and the c.m.s. energy $\sqrt{S}$ probes both the PDFs and the FFs at a different range of momentum fractions and scales of $\mathcal{O}\left(p_{T}\right)$. Likewise, different hadron species $H=\pi^{0}, \pi^{ \pm}$, $K^{ \pm}, \ldots$ determine complementary aspects of the hadronization process, i.e., different FFs $D_{c}^{H}$. The relevance of each parton flavor $c$ depends on the quark content of $H$ and on $p_{T}$ and $\sqrt{S}$, which control the contributions of the various partonic channels $a b \rightarrow c X$ to the sum in Eq. (1). We will demonstrate how the different partonic subprocesses are expected to contribute to hadron production yields at LHC energies.

A noteworthy property of the invariant cross section in Eq. (1) is its approximate power-law behavior $[12,13]$

$$
\sigma_{\mathrm{inv}} \equiv E \frac{d^{3} \sigma^{H}}{d \vec{p}}=F\left(x_{T}\right) / p_{T}^{n\left(x_{T}, \sqrt{S}\right)}
$$

for fixed $x_{T} \equiv 2 p_{T} / \sqrt{S}$. In the naive, scale-invariant parton model one expects scaling with $n=4$. The running of $\alpha_{s}$ and the scaling violations of the PDFs and FFs as predicted by pQCD lead to deviations from exact scaling for Eqs. (1) and (2), i.e., $n=n\left(x_{T}, \sqrt{S}\right)$, which can be explored by comparing, e.g., $x_{T}$ hadron spectra at different $\sqrt{S}$. We shall briefly touch upon $x_{T}$ scaling at LHC energies and estimate theoretical scale uncertainties in predicting $n=n\left(x_{T}, \sqrt{S}\right)$.

\section{B. Applicability of FFs and uncertainty estimates}

Since the LHC sets a new energy frontier for hadron production, it is crucial to first convince ourselves that the accessible range of $z$ is still compatible with the applicability of the concept of FFs [14] within the factorized framework as outlined above. Contrary to PDFs, where the small $x_{a, b}$ regime is rather well explored down to momentum fractions of $\mathcal{O}\left(10^{-4}\right)[2-4]$ and has been subjected to a very detailed theoretical scrutiny, the phenomenological access to FFs is much more restricted. Neglected hadron mass effects, potential higher twist corrections, and instabilities in the timelike scale evolution limit the usage of FFs to rather large values of $z \gtrsim 0.05$; see, e.g. Ref. [7]. We shall show that for all practical applications at the LHC the condition $z \gtrsim 0.05$ is well met as the bulk of the cross section for inclusive hadron production samples on average large momentum fractions, $\langle z\rangle \simeq 0.5$, well above the kinematic lower limit $z \approx 2 p_{T} / \sqrt{S}$.

Upcoming experimental results for single-inclusive hadron production at the LHC can be straightforwardly included [15] in existing global QCD analyses of FFs [7,8], which are the most efficient and consistent method to deconvolute information on these nonperturbative functions from the interplay of various observables measured at different energy scales. The large range of transverse momenta $p_{T}$ accessible at the LHC will allow for unprecedented studies of evolution effects for FFs. We note that for both the timelike scale evolution of FFs [16] and the partonic hard scattering cross sections $d \hat{\sigma}_{a b \rightarrow c X}$ [17] in Eq. (1), pQCD calculations at next-to-leading order (NLO) accuracy are "state of the art" and used throughout this work. In any case, they are mandatory for an accurate and meaningful comparison of theory and data due to often 
sizable NLO QCD corrections and, in particular, to allow for estimates of scale uncertainties.

As mentioned above, besides establishing a small set of "best-fit" parameters in a $\chi^{2}$ minimization to model the functional form of the FFs for different flavors and hadrons, assessing their uncertainties and propagating them to physical observables is an equally important goal. Here, the most robust technique is based on Lagrange multipliers [6-8] which makes no assumptions about the behavior of the $\chi^{2}$ profile near its minimum. We illustrate the usefulness of this method by estimating the uncertainties from FFs for charged hadron production at the LHC and Fermilab's Tevatron $p \bar{p}$ collider. For the latter, data from the CDF collaboration [18] have recently caused some stir as the measured cross section above $p_{T} \geq 20 \mathrm{GeV}$ exceeds theoretical expectations by orders of magnitude [19]. Since the data are also in excess of single-inclusive jet cross section measurements, the most likely explanation seems to be some experimental problem [19]. Nevertheless, we believe it is an useful exercise to evaluate the theoretical ambiguities caused by FFs also in this case. It turns out that naive estimates, for instance, by comparing the results obtained with two different sets of FFs, often seriously underestimate uncertainties from FFs.

\section{FFs in a nuclear environment at the LHC}

It is well known that results for hadron production processes occurring in a nuclear medium can differ significantly from similar experiments involving only light nuclei or proton targets, showing both suppression and enhancement of the rates depending on the details of the observable. Recent examples include production rates of pions and kaons in semi-inclusive deep-inelastic scattering (SIDIS) off different nuclei as provided by the HERMES experiment [20] and in deuteron-gold collisions measured at RHIC [21]. The origin of the observed nuclear modifications has been attributed to a variety of conceivable mechanisms and models [11] besides the well-known modification of parton densities in nuclei (nPDFs) [22-24]. Available models incorporate ideas based on interactions between the nuclear medium and, e.g., the final-state hadron or the seed partons before the hadronization takes place, and reproduce, with different degree of success, some features of the data; for recent reviews, see Ref. [11].

nPDFs provide an effective and phenomenologically successful way to factorize the influence of the nuclear environment on the interacting partons into sets of universal functions which scale in energy like ordinary PDFs and can be obtained in global QCD fits to available data [22-24]. The quite natural extension of this idea to finalstate nuclear effects has been put forward only very recently by introducing the concept of medium modified fragmentation functions (nFFs) [11]. First QCD fits for identified pions and kaons were provided in Ref. [25] recently. As for nPDFs, it was demonstrated that within the precision of the available data, universal nFFs are a viable concept and factorization similar to Eq. (1) holds at least approximately [25] despite being much more speculative than in $p p$ collisions $[11,26]$ due to, e.g., multiple gluon emission of the partons traversing the strong color field of the dense QCD medium. The nuclear $A$ dependence of the nFFs can be most economically parametrized in a convolution approach [25]

$$
D_{c / A}^{H}\left(z, \mu_{0}\right)=\int_{z}^{1} \frac{d y}{y} W_{c}^{H}\left(y, A, \mu_{0}\right) D_{c}^{H}\left(\frac{z}{y}, \mu_{0}\right),
$$

which relates the nFFs $D_{c / A}^{H}$ to the fairly well-known vacuum FFs of De Florian-Sassot-Stratmann (DSS) [7] at some initial scale $\mu_{0}$ through a weight function $W_{c}^{H}$ with only a small amount of extra parameters. nFFs at scales $\mu>\mu_{0}$ are then obtained by applying the standard timelike evolution equations [16].

Combined with nPDFs, nFFs allow one to treat a large class of hard hadron production processes where a nucleus collides with a lepton or a nucleon (light nucleus) in a consistent pQCD framework based on factorization. Exploiting the predictive power of the factorized approach, we will provide predictions for pion production in future proton-lead $(p P b)$ collisions at the LHC in a wide range of $p_{T}$ and rapidity. $p P b$ collisions at the LHC are conceivable up to a c.m.s. energy of $\sqrt{S} \approx 8.8 \mathrm{TeV}$ [27] though not part of the initial LHC physics program. In addition, we shall discuss how the admixture of the different contributing partonic subprocesses $a b \rightarrow c X$ and the range of probed momentum fractions $z$ is expected to change in a nuclear environment at LHC energies. Such measurements will be crucial to further our knowledge of hadronization in a nuclear medium by exploring to what extent factorization breaking effects due to interactions of partons with the medium come into play and limit the usefulness and predictive power of nPDFs and nFFs. Comparisons of hadron rates obtained $p p$ and $p P b$ collisions will also help to unravel and understand the properties of hot and dense QCD matter.

The remainder of the paper is organized as follows: in the next section, we compare first results for $p_{T}$ spectra of unidentified charged hadrons from ATLAS [28] and CMS [29] with pQCD calculations at NLO accuracy. In Sec. III, we present expectations for both identified and unidentified hadron production cross sections in $p p$ collisions at the LHC in a broad range of $p_{T}$ and rapidity $y$. The results are supplemented by studies of theoretical scale and PDF ambiguities, the relevance of different partonic subprocesses, and the ranges of momentum fractions $x_{a, b}$ and $z$ predominantly probed. We touch upon $x_{T}$ scaling and give estimates of FF uncertainties for charged particle yields at the LHC and the Tevatron using the Lagrange multiplier technique. Pion production in $p P b$ collisions and medium modified FFs are discussed in Sec. IV. We summarize our main results in Sec. V. 


\section{COMPARISON TO FIRST LHC DATA}

Before turning to a detailed discussion of theoretical expectations for single-inclusive hadron production at LHC energies in Sec. III, we take a brief look at first results from ATLAS [28], CMS [29], and ALICE [30] for $p_{T}$ differential charged hadron yields.

Charged hadron multiplicities are the first results of the LHC physics program, and data were reported shortly after the startup of the LHC by the ALICE [30,31], ATLAS [28], and CMS [29] experiments which impressively demonstrated the readiness of their detector systems. They provide measurements of the number of charged hadrons $N_{\mathrm{ch}}$ with respect to (w.r.t.) their transverse momentum $p_{T}$ and pseudorapidity $\eta$. The main result so far is the increase of the pseudorapidity density $d N_{c h} / d \eta$ at central rapidities with the c.m.s. energy of the $p p$ collisions in the range $\sqrt{S}=0.9-7 \mathrm{TeV}[29,31]$.

The pseudorapidity density $d N_{c h} / d \eta$ is not amenable to pQCD calculations based on factorization, Eq. (1), as the bulk of the produced hadrons has very low $p_{T}$, well below $1 \mathrm{GeV}$, and a hard scale is lacking. However, both ATLAS [28] and CMS [29] also present data for $d N_{\mathrm{ch}} / d p_{T}$ in the rapidity range $|\eta| \leq 2.5$ and $|\eta| \leq 2.4$, respectively, at sufficiently high $p_{T}$. Very recently, also ALICE published a $p_{T}$ spectrum at $\sqrt{S}=900 \mathrm{GeV}$ and $|\eta| \leq 0.8$ [30]. In each case, the charged particle multiplicities are normalized to the number of inelastic non-single-diffractive (NSD) interactions but based on slightly different event selections.

In order to compare the obtained invariant yields $1 /\left(2 \pi p_{T}\right) d^{2} N_{\mathrm{ch}} / d \eta d p_{T}$ with pQCD predictions for $E d^{3} \sigma^{H} / d \vec{p}$ in Eq. (1), one needs to normalize them with the total NSD cross section $\sigma_{\mathrm{NSD}}$, which unfortunately is not specified in Refs. [28-30]. However, we estimate $\sigma_{\mathrm{NSD}}$ for each available data set by scaling the theoretical singleinclusive hadron yields at NLO by $1 / \sigma_{\mathrm{NSD}}$ and fitting a common, i.e., $p_{T}$-independent, value for each experiment. We use the CTEQ6.6 set of PDFs [2] and the DSS partonto-unidentified charged hadron FFs [8] in Eq. (1) and choose $\mu_{f}=\mu_{f^{\prime}}=\mu_{r}=\mu=p_{T}$. The results of the NLO calculations are shown as solid lines in Figs. 1-3 and compared to ATLAS [28], CMS [29], and ALICE [30] data, respectively. Note that only data with $p_{T} \geq 1 \mathrm{GeV}$ are displayed. We refrain from giving theoretical expectations based on Eq. (1) for $p_{T} \lesssim 1.5 \mathrm{GeV}$ where power suppressed corrections to the factorized $\mathrm{pQCD}$ framework and other nonperturbative soft contributions are relevant. In any case, all sets of PDFs [2] and FFs [8] are not applicable for too small scales $\mu \simeq p_{T}$.

The shaded bands in Figs. 1-3 give an indication of the theoretical uncertainties due to the truncation of the perturbative series in Eq. (1) at NLO accuracy. As is customary, they are obtained by simultaneously varying all scales in Eq. (1) by a factor of 2 around the default choice $\mu_{r}=p_{T}$. Given the fact that the normalization of the

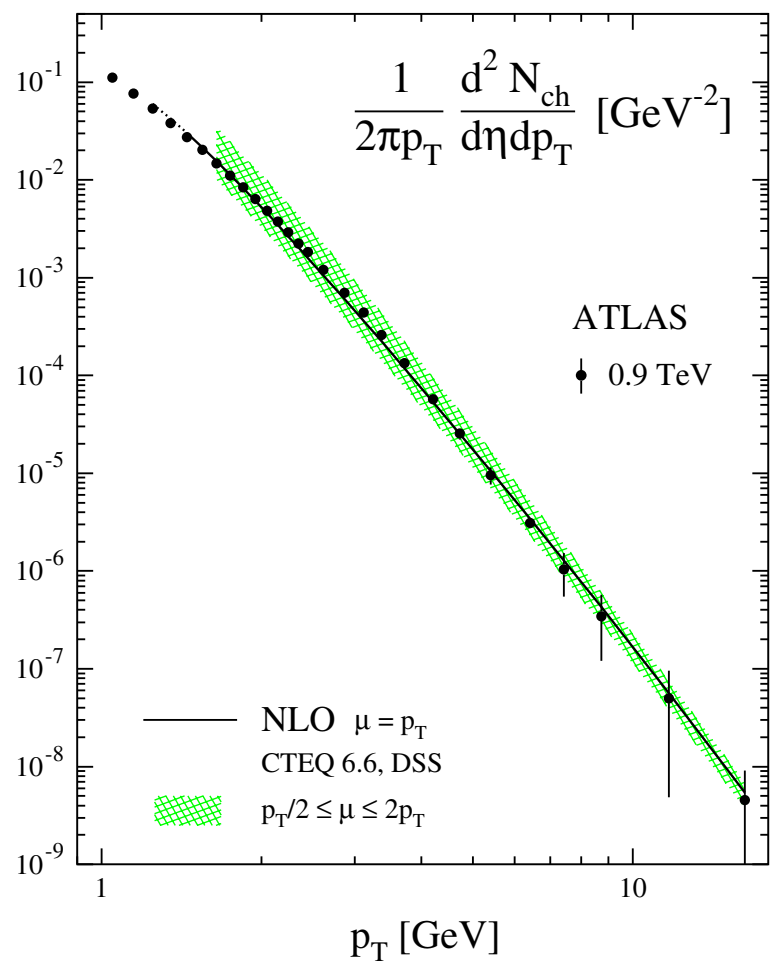

FIG. 1 (color online). Comparison of the single-inclusive charged hadron yield per NSD event in the rapidity range $|\eta| \leq$ 2.5 in $p p$ collisions at $\sqrt{S}=900 \mathrm{GeV}$ measured by ATLAS [28] with a NLO pQCD calculation using the DSS FFs [8] and CTEQ6.6 PDFs [2] (solid line). The shaded band indicates the theoretical uncertainty from varying the scales in Eq. (1) by a factor of 2 around the default choice $\mu_{f}=\mu_{f^{\prime}}=\mu_{r}=p_{T}$. Note that the normalization of the curve is determined by a fit; see text.

theoretical results has to be determined by a fit, we refrain from studying other, usually subleading, sources of uncertainties like variations of PDF sets, at this point.

The overall agreement between the $p_{T}$ dependence as predicted by $\mathrm{pQCD}$ calculations at NLO accuracy and data is excellent, and the drop of the cross sections with increasing $p_{T}$ over many orders of magnitude is accurately reproduced. As was already noticed in $p p$ collisions at BNL-RHIC at lower c.m.s. energies, NLO pQCD calculations at the leading-twist level tend to describe singleinclusive hadron yields well down to fairly low values of $p_{T} \simeq 1.5 \mathrm{GeV}$. We note a mild tension with CMS data [29] in Fig. 2 for $\sqrt{S}=7 \mathrm{TeV}$ and $p_{T} \geqslant 4.5 \mathrm{GeV}$, which are all at the lower edge of the scale uncertainty band. Notice that of all hadron production data, the ones taken at $\sqrt{S}=7 \mathrm{TeV}$ probe the smallest $x_{a, b}$ values in the PDFs so far, down to a kinematic limit of about $x_{T} \simeq 5 \times 10^{-4}$.

Upcoming precision data from the LHC will decisively test all theoretical aspects of inclusive hadron production in an unprecedented energy range, from nonperturbative PDFs and FFs to the validity of the leading-twist approximation in Eq. (1). In addition to unidentified charged 


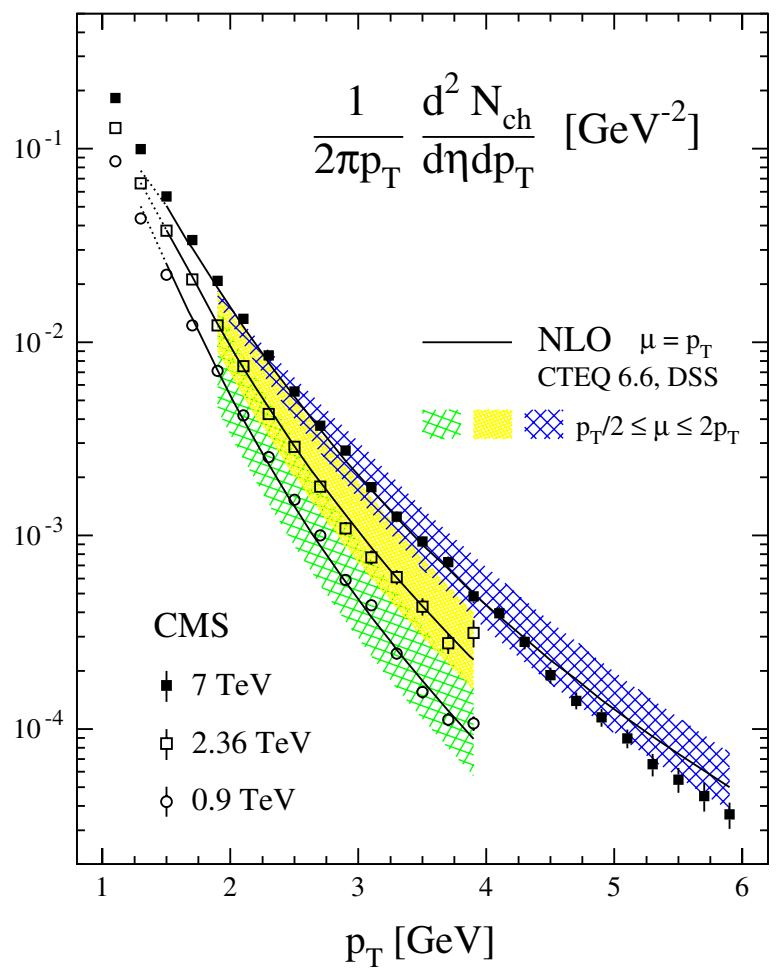

FIG. 2 (color online). As in Fig. 1 but now in the rapidity range $|\eta| \leq 2.4$ and for three different c.m.s. energies $\sqrt{S}=0.9,2.36$, and $7 \mathrm{TeV}$ as measured by the CMS experiment [29].

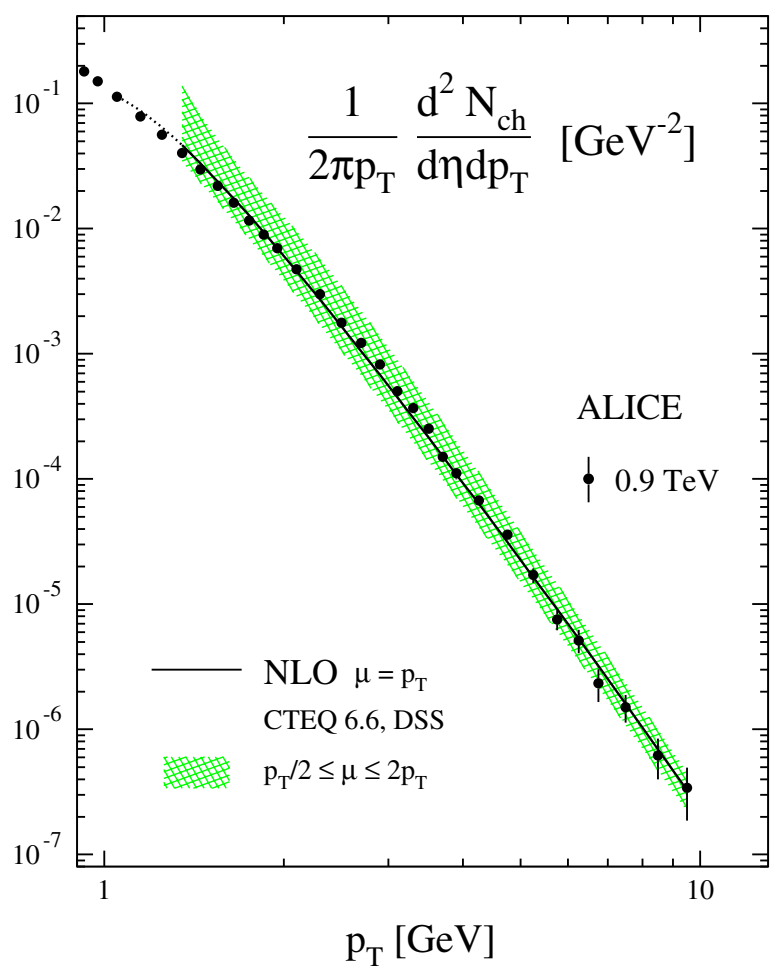

FIG. 3 (color online). As in Fig. 1 but now in the rapidity range $|\eta| \leq 0.8$ as measured by the ALICE experiment [30]. hadron yields measured so far, the ALICE experiment is designed to identify various hadron species like pions, kaons, and protons at central rapidities. These results will provide a vital input for future global analyses of FFs. Detailed theoretical expectations for single-inclusive hadron production at the LHC will be discussed in the next Section along with estimates of uncertainties.

\section{EXPECTATIONS FOR SINGLE-INCLUSIVE HIGH- $p_{T}$ HADRON PRODUCTION IN $p p$ COLLISIONS AT TEV ENERGIES}

Encouraged by the first results from the LHC experiments and the successful comparison to theoretical expectations based on the DSS set of fragmentation functions $[7,8]$, we now turn to a detailed discussion of both identified and unidentified hadron production cross sections to be studied at the LHC in the future. While ATLAS, CMS, and ALICE can all measure unidentified charged hadron yields, only ALICE is capable of tagging different hadron species at central rapidities, which is crucial for detailed tests of our current knowledge of FFs and the underlying framework of pQCD.

Our results are supplemented by estimates of theoretical scale, PDF, and FF uncertainties. To elucidate the impact of future LHC data on our understanding of $\mathrm{pQCD}$ factorization and, in particular, hadronization, we discuss the relevance of different partonic subprocesses $d \sigma_{a b \rightarrow c X}$ in Eq. (1), the role of quark and gluon fragmentation, and the ranges of momentum fractions which will be predominantly probed.

If not stated otherwise, we use the CTEQ6.6 set of PDFs and the associated Hessian sets for PDF uncertainty estimates [2]. The parton-to-hadron FFs are taken from DSS $[7,8]$, and uncertainties related to FFs will be estimated with the Lagrange multiplier method [6-8]. All calculations are performed at NLO accuracy with hard scattering cross sections, PDFs, and FFs in Eq. (1) evaluated in the $\overline{\mathrm{MS}}$ scheme. We take the transverse momentum $p_{T}$ of the produced hadron as the default choice for the factorization and renormalization scales, $\mu_{f, f^{\prime}}$ and $\mu_{r}$, respectively, in Eq. (1). To estimate theoretical ambiguities associated with the truncation of the perturbative series at NLO accuracy we vary all scales by a factor of 2 up and down their central value $p_{T}$ as is commonly done.

\section{A. Pion, kaon, and proton production at the LHC}

We start off with showing the energy dependence of the $p_{T}$ differential cross section for charged pion production integrated in the pseudorapidity range $|\eta| \leq 1.5$ in the upper panel of Fig. 4 for $\sqrt{S}=0.9,2.36,7$, and $14 \mathrm{TeV}$. The shaded bands give an estimate of the theoretical ambiguities due the variations of $\mu_{f, f^{\prime}, r}$ as described above. The typical scale uncertainty for $\sqrt{S}=14 \mathrm{TeV}$ amounts to about $25 \%$ at $p_{T} \approx 20 \mathrm{GeV}$ and $14 \%$ at $p_{T} \approx 100 \mathrm{GeV}$. 


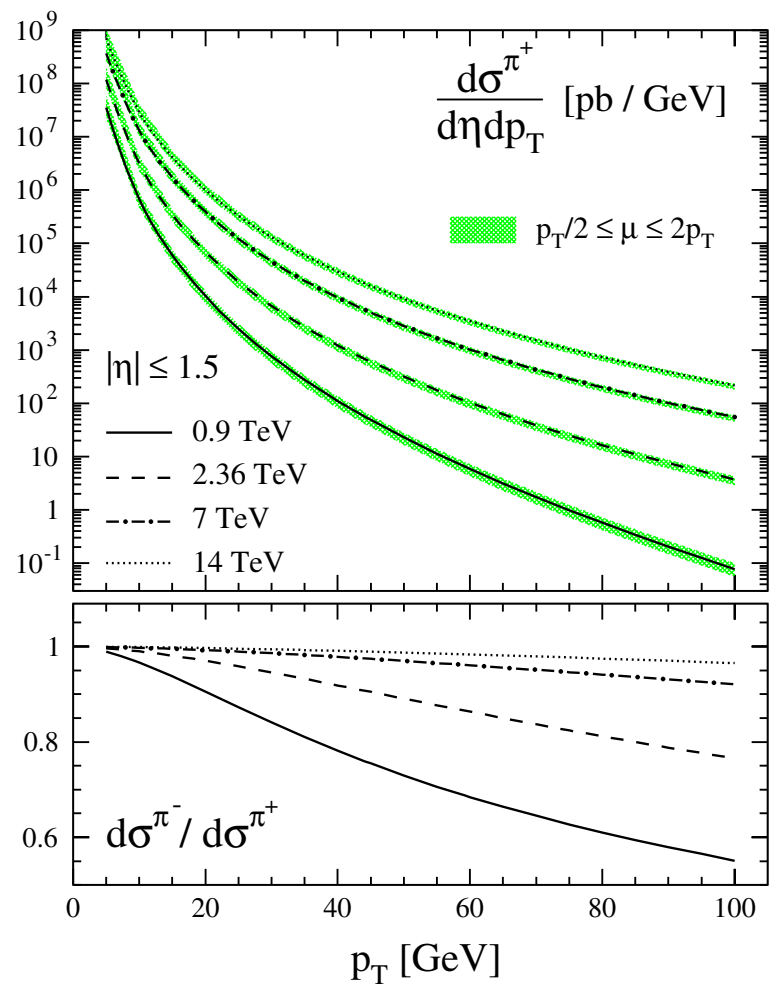

FIG. 4 (color online). Upper panel: NLO cross section for $\pi^{+}$ production at four different c.m.s. energies, integrated in the pseudorapidity range $|\eta| \leq 1.5$. The shaded bands indicate the uncertainty associated with the variation of the scales in the range $p_{T} / 2 \leq \mu_{f, f^{\prime}, r} \leq 2 p_{T}$. Lower panel: ratio of the $\pi^{-}$ and $\pi^{+}$yields.

At the same values of $p_{T}$ but lower c.m.s. energies, $\sqrt{S}=0.9 \mathrm{TeV}$, the corresponding uncertainties can reach up to $30 \%$.

The lower panel shows the expected difference of $\pi^{+}$ and $\pi^{-}$yields as predicted by the DSS set of FFs [7]. We recall that at present, the charge separation of FFs is mainly constrained by SIDIS data at a relatively low scale $Q^{2} \simeq$ $2.5 \mathrm{GeV}$ and to a much lesser extent by data from RHIC [7]. As a consequence, uncertainties are still sizable in current sets of FFs. As can be seen from Fig. 4, the ratio $d \sigma^{\pi^{-}} / d \sigma^{\pi^{+}}$drops only very slowly with increasing $p_{T}$ for both $\sqrt{S}=14$ and $7 \mathrm{TeV}$. This is due to the dominance of gluon initiated hard scattering and hadronization processes, which prevails up to fairly high $p_{T}$; see Figs. 7(a) and 7 (c) and the discussions below. Clearly, a precise measurement of $d \sigma^{\pi^{-}} / d \sigma^{\pi^{+}}$is of great phenomenological importance but at the same time also very challenging at nominal LHC energy as sufficiently large integrated luminosities are required to resolve effects of a few percent at high $p_{T}$. Lower c.m.s. energies, $\sqrt{S}=2.36$ and $0.9 \mathrm{TeV}$ are more favorable as they probe larger momentum fractions $x_{a, b}$ in the nucleon in the same range of $p_{T}$, i.e., larger $x_{T} \equiv$ $2 p_{T} / \sqrt{S}$, where quark initiated scattering processes more and more dominate.
The expectation that $d \sigma^{\pi^{+}}>d \sigma^{\pi^{-}}$can be understood by inspecting the role of different partonic subprocesses in $p p$ collisions; see Fig. 7(a) below. Quark-gluon scattering is the second most important channel for hadron production. The abundance of $u$ quarks in a proton and the $u \bar{d}$ valence flavor structure of a $\pi^{+}$, i.e., $D_{u}^{\pi^{+}}>D_{u}^{\pi^{-}}$, explains the observed hierarchy for the $\pi^{ \pm}$production yields found in Fig. 4.

Figure 5 compares the cross sections for $\pi^{+}, K^{+}$, and proton production as a function of $p_{T}$ for $\sqrt{S}=7 \mathrm{TeV}$ and $\eta=0$. At $p_{T} \approx 20 \mathrm{GeV}$ pions yields are roughly a factor of 3 larger than those for $K^{+}$, and the production of protons is suppressed even further. Together, pions, kaons, and protons account for almost the entire yield of charged hadrons; see Fig. 9 below. The mixture of hadron species is largely independent of $p_{T}$, with the fraction of pions decreasing slightly as $p_{T}$ increases. This is because the mean value of $z$ changes only very slowly with $p_{T}$, see Fig. 7(e) below. At higher $z$, fragmentation into heavier hadrons like kaons and protons is somewhat enhanced w.r.t. those into pions $[7,8]$.

The typical PDF uncertainty for hadron production at the LHC is illustrated in the lower panel of Fig. 5 and

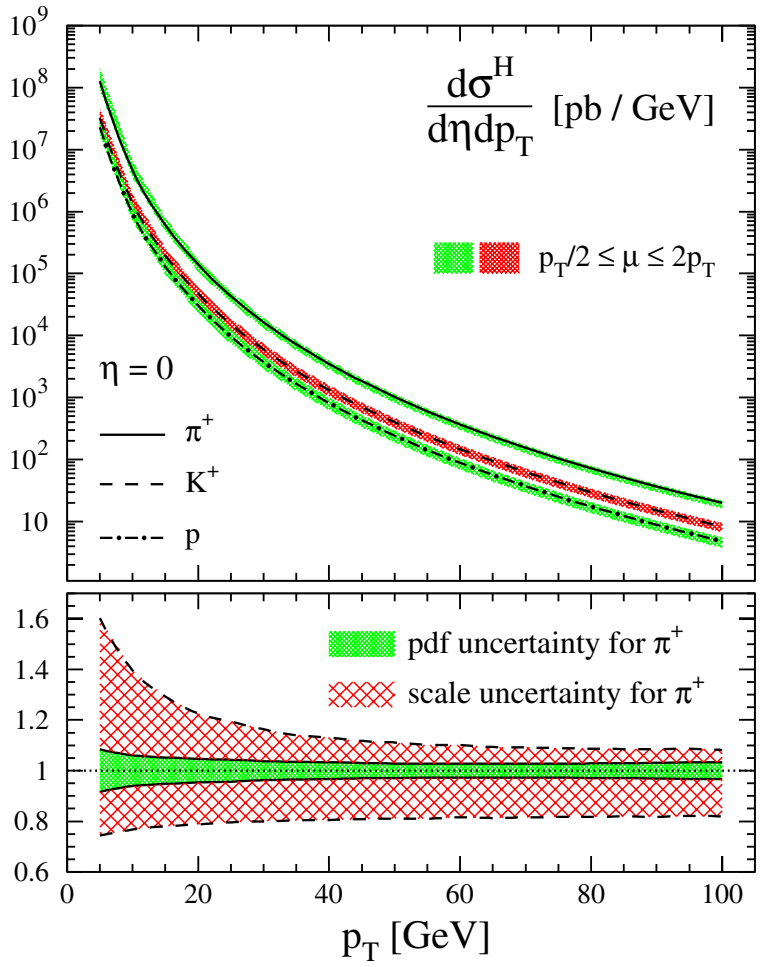

FIG. 5 (color online). Upper panel: comparison of the yields for $\pi^{+}, K^{+}$, and $p$ at NLO accuracy for pseudorapidity $\eta=0$ and $\sqrt{S}=7 \mathrm{TeV}$. As before, the shaded bands give an estimate of the theoretical scale ambiguity. Lower panel: PDF uncertainty for $\pi^{+}$production w.r.t. the solid line in the upper panel, estimated with the Hessian eigenvector sets for CTEQ6.6 [2] (solid band) and compared to the ambiguity from scale variations (hatched band). 
amounts to about $5 \%$ at $p_{T}=20 \mathrm{GeV}$. It is computed with the help of the 44 Hessian eigenvector sets provided by CTEQ [2] and compared to variations of the cross section due to the choice of scales $\mu_{f, f^{\prime}, r}$ in Eq. (1). Because of the dominance of gluon initiated scattering processes, see Fig. 7(a) below, the PDF uncertainty reflects to a large extent the present ambiguity in the gluon PDF at the relevant scale $\mu \simeq p_{T}$ and range of momentum fraction $x$. In particular at smaller values of $p_{T}$, the theoretical ambiguity due to the truncation of the $\mathrm{PQCD}$ series at NLO turns out to be by far the most relevant one.

The pseudorapidity dependence of the production cross section for neutral pions at $\sqrt{S}=7 \mathrm{TeV}$ is shown in Fig. 6 for various fixed values of $p_{T}$. Again, the typical scale uncertainty is indicated by the shaded bands for $p_{T}=10$ and $50 \mathrm{GeV}$. Measurements of the $\eta$ dependence for fixed $p_{T}$ in a wide range are of great phenomenological interest as they emphasize different partonic subprocesses and momentum fractions $x_{a, b}$ and $z$ as compared to the $p_{T}$ differential yields shown in Figs. 4 and 5.

These aspects are explained in some detail in Fig. 7. The panels show the relevance of the different NLO partonic subprocesses $d \sigma_{a b}$ (upper row), the relative fractions of quark and gluon fragmentation (middle row), and the mean values of the momentum fractions $x_{a, b}$ and $z$ (lower row) probed in $\pi^{0}$ production at $\sqrt{S}=7 \mathrm{TeV}$. The results on the

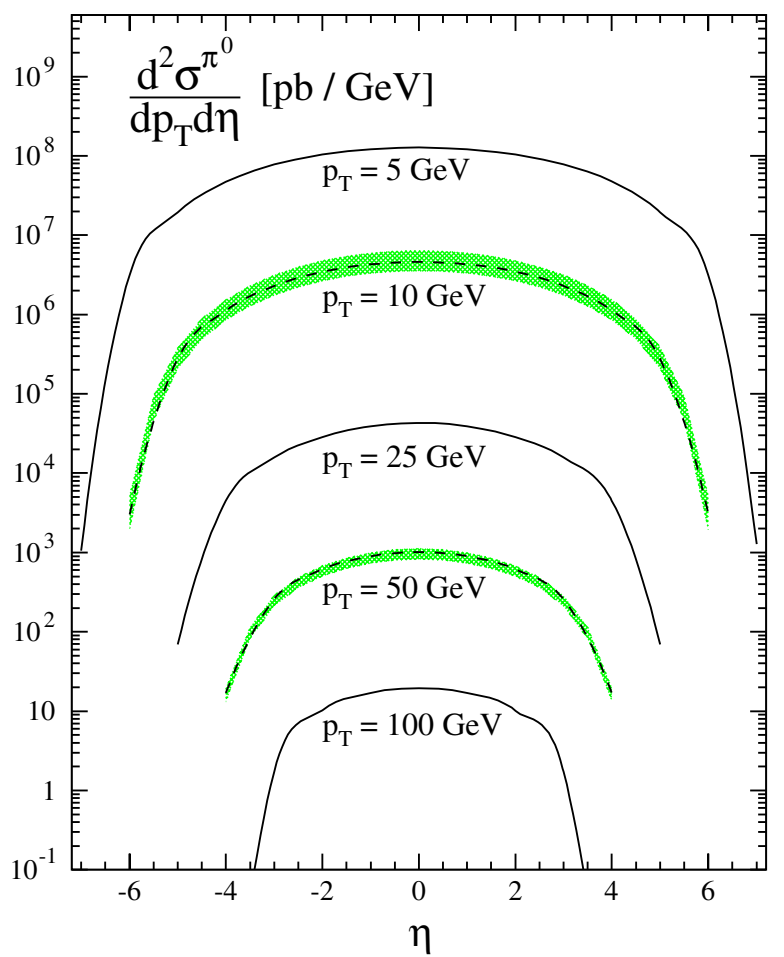

FIG. 6 (color online). Pseudorapidity dependence of the neutral pion production cross section at $\sqrt{S}=7 \mathrm{TeV}$ for various fixed values of $p_{T}$. The typical theoretical scale uncertainty is indicated by the shaded bands for $p_{T}=10$ and $50 \mathrm{TeV}$.

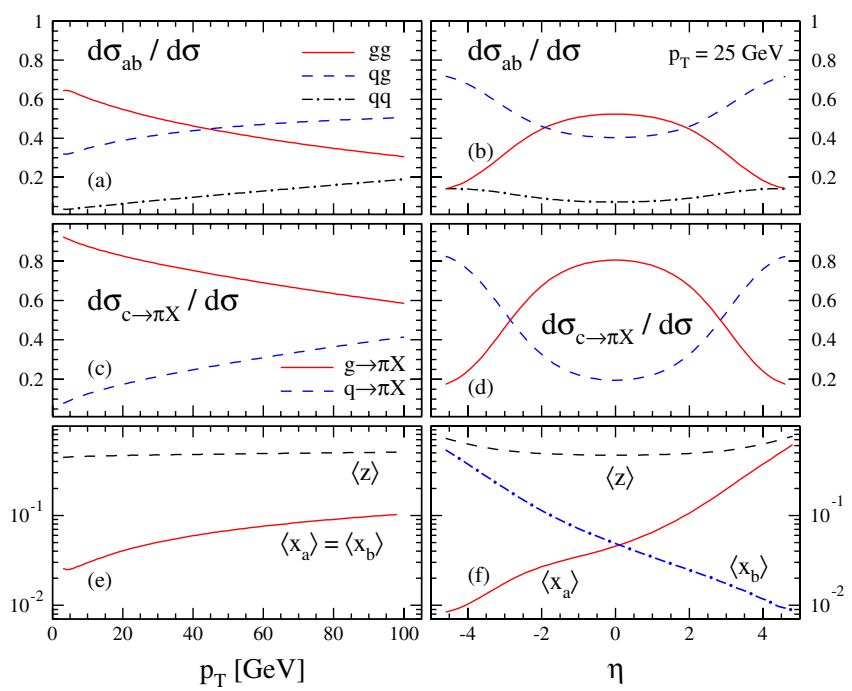

FIG. 7 (color online). (a) and (b): relative contributions of NLO partonic subprocesses $d \sigma_{a b}$ initiated by gluon-gluon, quark-gluon, and quark-quark scattering to the $\pi^{0}$ cross section at $\sqrt{S}=7 \mathrm{TeV}$ as a function of $p_{T}$ and $\eta$, respectively. (c) and (d): same as in (a) and (b) but now for the relative fractions of quarks and gluons fragmenting into the observed pion. (e) and (f): mean values of the momentum fractions $x_{a, b}$ and $z$ probed in $\pi^{0}$ production.

left-hand side are given as a function of $p_{T}$ for $\eta=0$, and the panels on the right-hand side are differential in $\eta$ for fixed $p_{T}=25 \mathrm{GeV}$. For different c.m.s. energies, the plots roughly scale with $x_{T}=2 p_{T} / \sqrt{S}$, i.e., subprocess fractions are similar for the same value of $x_{T}$.

From panels (a) and (b) one can infer that gluon-gluon scattering is the most important channel at relatively small $p_{T}$ and central rapidities $\eta$. For $p_{T} \gtrsim 40 \mathrm{GeV}$ at $\eta \simeq 0$ or at forward rapidities, quark-gluon scattering becomes the dominant subprocess. For instance, at large forward rapidities, $\eta \gg 0$, one is mainly sensitive to the scattering of a valence quark carrying a large momentum fraction $x_{a}$ off a gluon with $x_{b} \ll 1$ for which both PDFs are large; cf. also panel (f). In the shown kinematic range, quark-quark initiated processes are always small and reach a level of $15 \%$ only for large $p_{T}$ and/or large pseudorapidities $\eta$.

Figures 7(c) and 7(d) give the relative contributions of quark and gluon fragmentation to the $p_{T}$ and $\eta$ differential cross sections, respectively. At mid rapidity, gluon-to-pion fragmentation is dominant in the entire range of $p_{T}$ shown. For $|\eta| \gg 0$ and $p_{T}=25 \mathrm{GeV}$, quark fragmentation reaches a level of $80 \%$. We note that for smaller values of $p_{T}$, the share between quark and gluon induced hadron production is roughly equal.

In the lower panels of Fig. 7 we present estimates of the mean momentum fractions $\left\langle x_{a, b}\right\rangle$ and $\langle z\rangle$ which are predominantly probed in single-inclusive hadron production at the LHC. There are several ways to estimate, for instance, an average $\langle z\rangle$. We define it in the standard way by evaluating the convolutions in Eq. (1) with an extra factor 
of $z$ in the integrand, divided by the cross section itself [32], i.e., schematically we use

$$
\langle z\rangle \equiv \frac{\int d z z \frac{d \sigma^{H}}{d z d p_{T}}}{\int d z \frac{d \sigma^{H}}{d z d p_{T}}} .
$$

$\left\langle x_{a, b}\right\rangle$ are estimated accordingly. The most important thing to notice is that despite the large c.m.s. energies available at the LHC, the bulk of the hadrons is produced with $\langle z\rangle \geq 0.4$ and $\langle z\rangle \geq 0.6$ for $\eta=0$ and $\eta \gg 1$, respectively, which is comparable to what one finds at much lower c.m.s. energies, for instance, at RHIC. This important finding ensures the applicability of the concept of FFs which, as discussed in the Introduction, requires $z \geq 0.05$. Another interesting result is that at mid rapidity the average $x_{a, b}$ is considerably larger than what one might naively expect from the lower kinematic limit $x_{a, b} \simeq x_{T}=$ $2 p_{T} / \sqrt{S}$ which is of the order a few times $10^{-3}$ for $p_{T} \lesssim$ $20 \mathrm{GeV}$.

Figures 4-7 clearly demonstrate the potential impact of single-inclusive hadron measurements in a wide kinematic range in $p_{T}$ and $\eta$ on future FF global analyses. Data will impose stringent constraints on the FFs for various hadron species in the large $z$ range at unprecedented large scales $\mu_{f^{\prime}} \simeq p_{T}$. Scale and to some extent also PDF uncertainties are considerably smaller than for corresponding measurements at RHIC performed at considerably smaller values of $p_{T}$ and $\sqrt{S}$ which are currently used in global fits $[7,8]$.

Finally, we discuss the property of $x_{T}$ scaling at LHC energies. In $\mathrm{pQCD}$ one expects deviations from the naive power-law scaling of the invariant cross section $\sigma_{\text {inv }}$ in Eq. (2) leading to $n=n\left(x_{T}, \sqrt{S}\right)[12,13]$. Figures 8 (a) and 8(b) show ratios of $\sigma_{\text {inv }}$ scaled by $p_{T}^{n}$ for $7 \mathrm{TeV}, 2.36 \mathrm{TeV}$, and $0.9 \mathrm{TeV}$ to the result obtained for $\sqrt{S}=14 \mathrm{TeV}$ for fixed $n=4$ and $n=5$ in Eq. (2), respectively. As can be seen, scaling violations are sizable for $n=4$ as should be expected from the running of $\alpha_{s}$ and the scale evolution of PDFs and FFs. Note that the ratio for $0.9 \mathrm{TeV}$ is too large to be displayed in Fig. 8(a). The choice $n=5$ in Eq. (2) leads to much more similar ratios for all three energies in the entire range of $x_{T}$, in particular, for $\sqrt{S}=2.36 \mathrm{TeV}$ and $0.9 \mathrm{TeV}$.

Power-law scaling of $\sigma_{\text {inv }}$ with a universal exponent $n$ can, however, never be more than a rough approximation because $n$ must depend on both $p_{T}$ and $\sqrt{S}$ rather than being constant. This kinematic dependence of $n$ is illustrated in Fig. 8(c) for two different values of $p_{T}$. As in Ref. [13], we estimate the scaling exponent $n$ in Eq. (2) by comparing $x_{T}$ spectra at different c.m.s. energies $\sqrt{S}$ and $\sqrt{S^{\prime}}$ for fixed $p_{T}$, i.e.,

$$
n\left(x_{T}\right)=-\frac{\ln \left[\sigma_{\text {inv }}\left(S, x_{T}\right) / \sigma_{\text {inv }}\left(S^{\prime}, x_{T}\right)\right]}{\ln \left(\sqrt{S} / \sqrt{S^{\prime}}\right)} .
$$

A dependence on both $x_{T}$ and $p_{T}$ is clearly visible and needs to be taken into account when comparing theoretical

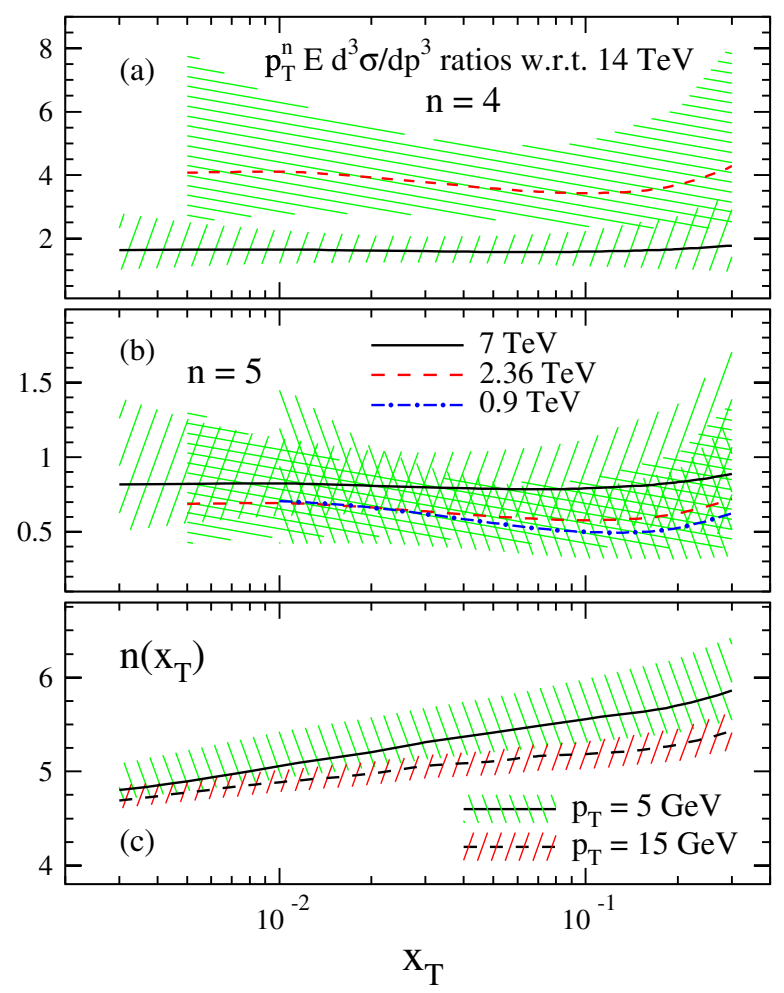

FIG. 8 (color online). Illustration of $x_{T}$ scaling for $\pi^{+}$production at $\eta=0$ and LHC energies. (a) and (b): ratios of the invariant cross section in Eq. (2) scaled by $p_{T}^{n}$ for $7 \mathrm{TeV}$ (solid line), $2.36 \mathrm{TeV}$ (dashed line), and $0.9 \mathrm{TeV}$ (dot-dashed line) to the result obtained for $\sqrt{S}=14 \mathrm{TeV}$ for $n=4$ and $n=5$, respectively. The bands indicate the theoretical uncertainty from varying the scales $\mu_{f, f^{\prime}, r}$ independently in the numerator and denominator of the ratios. (c): $x_{T}$ dependence of the scaling exponent $n$ and scale uncertainty (shaded bands) estimated based on Eq. (5).

expectations for $x_{T}$ scaling to experimental spectra. Consequently, there is also some ambiguity in estimating $n\left(x_{T}\right)$ as the result based on Eq. (5) depends also on the choice of $S$ and $S^{\prime}$.

In addition, studies of the scaling behavior also suffer, of course, from theoretical scale ambiguities. The shaded bands in Figs. 8(a) and 8(b) indicate the uncertainty from varying the scales $\mu_{f, f^{\prime}, r}$ by the usual factor of 2 up and down the default choice $p_{T}$. Compared to Ref. [13], we find a much larger variation because, as a more conservative choice, we allow for different scales in the numerator and denominator of the ratios of invariant cross sections shown in panels (a) and (b).

The possibility of having different factorization scales for observables calculated at different c.m.s. energies is, e.g., natural in certain proposed "scale fixing" procedures [33], as the "optimum scale" depends on the kinematics of the process. It is also interesting to recall recent measurements of single-inclusive neutral pion production at RHIC for three different c.m.s. energies [34]. Although the cross sections are described well by $\mathrm{pQCD}$ within the sizable 
scale uncertainties, it can be argued that an "optimized" choice of scales in a NLO calculation [17] would suggest to use scales closer to $p_{T} / 2, p_{T}$, and $2 p_{T}$ for $\sqrt{S}=62.4$, 200 , and $500 \mathrm{GeV}$, respectively. The smaller factorization scale at lower c.m.s. energies allows for more QCD radiation in the hard scattering matrix elements which increases the cross section and leads to a more favorable description of the data. To some extent this mimics all order resummations of logarithmic contributions in the partonic subprocess cross sections which are enhanced near the partonic threshold and hence more relevant at lower c.m.s. energies for a given value of $p_{T}$ [35].

\section{B. Unidentified charged hadron spectra}

In the following, we provide a detailed assessment of how the present limitations of our knowledge of FFs propagate to uncertainties for cross section estimates. We focus the discussions on unidentified charged hadron spectra, which, in addition to be accessible with all LHC detectors as well as the Tevatron experiments, allow us to compare the relative contributions of different hadron species and their respective uncertainties.

Pions clearly dominate the charged hadron spectra, with typical uncertainties for the relevant FFs $D_{c}^{\pi^{ \pm}}$estimated to be at the few percent level [7]. Nevertheless, charged kaons, protons, and antiprotons represent non-negligible contributions and, potentiated by the much larger uncertainties inherent to their FFs [7,8], may yield a significant contribution to the theoretical error for estimates of charged particle spectra, perhaps even comparable in size to the one for pions. Contributions from "residual charged hadrons", i.e., hadrons other than pions, kaons, and protons, are known to be completely marginal at low energy scales but increase with energy. Since the corresponding FFs are very poorly known [8], they can produce sizable uncertainties, comparable or even larger than those coming from the much more copiously produced hadron species. Altogether, the combined theoretical error from FFs may become comparable to scale uncertainties in certain kinematic regions, in particular, at larger transverse momenta.

To a first approximation, uncertainties from FFs are often estimated by simply comparing the results obtained with two different optimum fits of FFs. Such a procedure can give, however, at best a lower bound on the true error. In order to arrive at a faithful estimate of uncertainties derived from those inherent to FFs, we use the robust Lagrange multiplier technique [6], which explores for any desired observable depending on FFs its full range of variations within chosen the range of $\Delta \chi^{2}$ tolerated in the fit. Although the actual error estimate is more involved than in the standard Hessian method [5], it has the advantage of not making any assumptions on the shape of the $\chi^{2}$ profile near the minimum of the fit or on how the errors of the fit parameters describing the FFs propagate to a given observable.
As an example, the upper right panels of Figs. 9 and 10 show the outcome of propagating the uncertainties of the FFs for different hadron species with the Lagrange multiplier method to the invariant charged hadron cross sections (given in the left panels) for both the LHC and the Tevatron at c.m.s. energies of 7 and $1.96 \mathrm{TeV}$, respectively. As in the DSS analysis [7,8], we allow for a tolerance of $\Delta \chi^{2} /$ $\chi^{2}=2 \%$ in each error analysis. The uncertainties resulting from FFs are compared to the theoretical ambiguity from variations of the scales within the typical range $p_{T} / 2 \leq$ $\mu_{f, f^{\prime}, r} \leq 2 p_{T}$. Although the scale uncertainties are dominant, errors propagated from FFs to the invariant charged hadron cross sections are quite sizable, and their role becomes increasingly significant at larger values of $p_{T}$. This is similar to what was found in Fig. 5 for PDF uncertainties.

In the lower right panels of Figs. 9 and 10 we show the relative contributions of the different hadronic species to the inclusive charged hadron spectrum as a function of $p_{T}$. It is worth noticing that the mixture of hadrons is fairly independent of $p_{T}$, which is linked to constant average momentum fraction $\langle z\rangle$ observed in Fig. 7(e). However, the contribution from residual charged hadrons becomes increasingly relevant at larger values of $p_{T}$ but still remains fairly small as compared to pions, kaons, and protons.

In Fig. 10 we compare the estimates for invariant cross section at NLO accuracy to recent data from CDF [18]. In the lower left panel we also show the ratio of data and theory. As can be seen, the theoretical results significantly undershoot the data for $p_{T} \gtrsim 25 \mathrm{GeV}$ which caused quite a

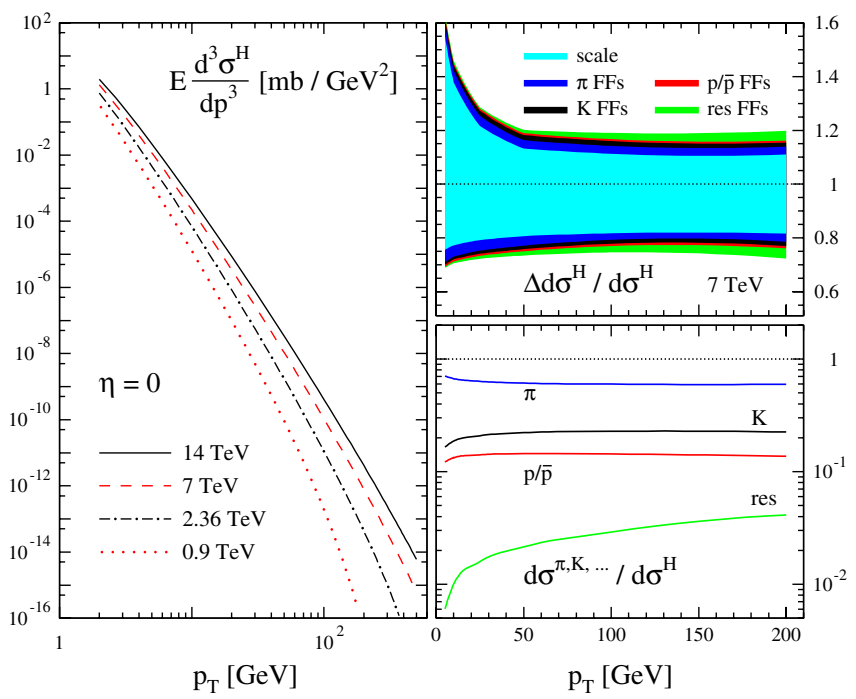

FIG. 9 (color online). Left panel: NLO invariant cross section for the production of charged hadrons for $\eta=0$ at four different c.m.s. energies. Upper right panel: relative theoretical ambiguity of the charged hadron yield at $\sqrt{S}=7 \mathrm{TeV}$ induced by variations of the scales $\mu_{f, f^{\prime}, r}$ and the uncertainties of the FFs for the individual hadron species. Lower right panel: Partial contribution of the different hadronic species to the invariant charged hadron cross section at $\sqrt{S}=7 \mathrm{TeV}$. 


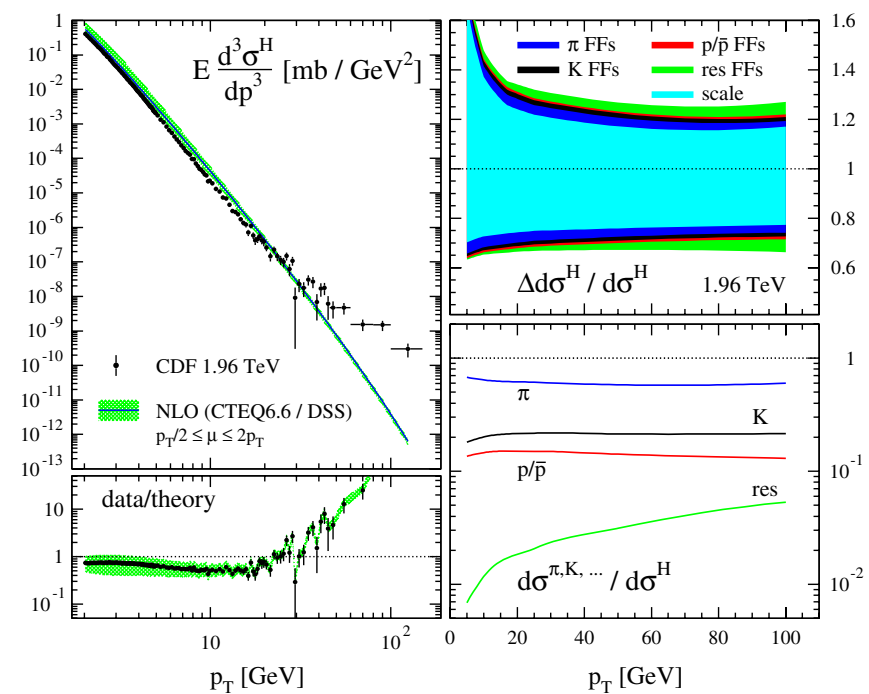

FIG. 10 (color online). The same as Fig. 9 but now for $p \bar{p}$ collisions at $1.96 \mathrm{TeV}$. The left panels show also recent data from CDF [18] and the ratio of data and a NLO calculation.

stir [19]. Since the data are also in excess of the corresponding jet measurement, the most likely explanation for the observed discrepancy is an experimental problem. Nevertheless, it is interesting to know that the FF uncertainties estimated above are by far too small to account for the hadron yield observed by CDF.

Finally, it is worth mentioning that when going from Tevatron to LHC kinematics there is quite some reduction in the relative importance of the residual factorization and renormalization scale dependence as compared to FF uncertainties. This again illustrates the impact of upcoming LHC data on future global analyses.

\section{PION PRODUCTION IN $p P b$ COLLISIONS}

In this section we propose a set of hadron production measurements in $p P b$ collisions at the LHC as a tool to characterize and quantify nuclear modifications in the fragmentation process. The LHC is capable of providing $p P b$ collisions at a maximum c.m.s. energy of about $8.8 \mathrm{TeV}$ [27] although such a program is not envisioned in the initial phase. However, measurements in $d A u$ collisions at RHIC were instrumental in interpreting results obtained in heavy ion collisions, which are considerably more complicated to understand theoretically.

We note that leading order estimates of neutral pion production in $p P b$ collisions at the LHC were recently presented in Ref. [36] but based on the assumption that all nuclear modifications can be entirely absorbed into nPDFs. In view of the known sizable medium induced effects on hadron production yields in, e.g., lepton-nucleus collisions [20], which cannot be explained by nPDFs [25], such an approach is questionable and may not be adequate. In fact, in a recent paper [25] the concept of medium modified fragmentation functions (nFFs) was introduced within the standard factorized framework of pQCD. These novel nFFs, which obey ordinary timelike scale evolution [16] and have been extracted in a global QCD analysis for pions and kaons, allow one to treat hard reactions with identified hadrons consistently at NLO accuracy when combined with nPDFs. This is the approach we pursue here to compute predictions for $p P b$ collisions at the LHC.

Conventional factorization of short and long-distance physics effects is, however, not expected to hold in general in a nuclear environment $[11,26]$. Quarks and gluons produced in the hard interaction may experience multiple scattering in the presence of the strong QCD color field of the dense nuclear medium which should affect, at least in principle, the fragmentation process. A well studied consequence of induced multiple gluon emissions would be an energy loss of the partons traversing the medium, see, e.g., Ref. [11] and references therein. This effect might be responsible for the observed strong suppression of singleinclusive hadron spectra in central collisions of two heavy nuclei w.r.t. properly scaled $p p$ interactions, a phenomenon often referred to as "jet quenching." The remarkable absence of a similar suppression of hadrons in $d A u$ collisions [21] is an indication that final-state interactions are the most relevant mechanism to explain these observations. Converting part of the energy of a produced hard parton into many soft partons, for instance, by multiple gluon emission, may alter also the QCD scale evolution of FFs depending on the properties of the medium, effectively breaking factorization. Several model calculations at treelevel accuracy have been proposed in the past years [26] based on different approximations and assumptions to account for multiple gluon emissions, the dynamical expansion of the medium, and possible coherence and geometrical effects.

It is of topical interest to understand quantitatively to what extent these effects induced by the medium are universal and can be mapped into $A$ dependent nFFs by choosing an appropriate weight function $W_{c}^{H}$ in Eq. (3), hence preserving the predictive power of QCD factorization for large $p_{T}$ processes at least at an approximative level. Recently, a global fit of nFFs based on the concept of QCD factorization [25] was shown to be phenomenologically very successful in describing current lepton-nuclei and deuteron-nuclei collision data, including their $A$ dependence [25]. This supports the concept of universal, medium modified PDF and FFs at least at an approximate level. $p P b$ collisions at the LHC will certainly explore the limits of characterizing nuclear modifications in a factorized pQCD approach.

To recall the pattern and magnitude of observed medium induced modifications for quarks and gluons, we show in Fig. 11 the ratios of nPDFs and nFFs

$$
R_{i}^{A}\left(x, Q^{2}\right) \equiv \frac{f_{i}^{A}\left(x, Q^{2}\right)}{f_{i}^{p}\left(x, Q^{2}\right)}, \quad R_{i / A}^{H}\left(z, Q^{2}\right) \equiv \frac{D_{i / A}^{H}\left(z, Q^{2}\right)}{D_{i}^{H}\left(z, Q^{2}\right)}
$$




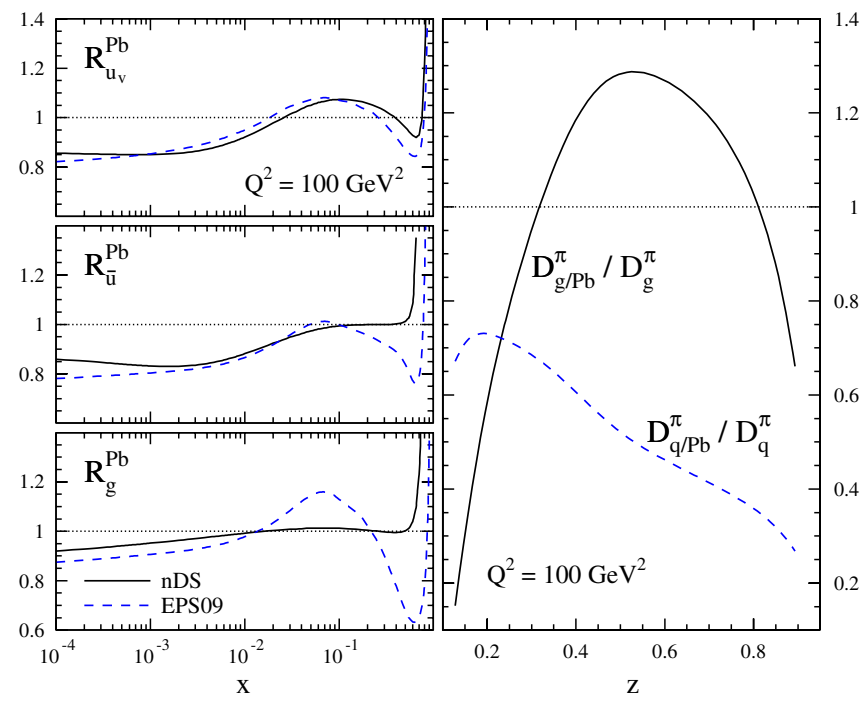

FIG. 11 (color online). Nuclear modification factors in lead for PDFs [22,24] (left panels) and FFs [25] (right panel) of different flavors at $Q^{2}=100 \mathrm{GeV}^{2}$ as determined in recent global QCD analyses at NLO accuracy.

to the standard PDFs and vacuum pion FFs, respectively. The ratios are evaluated for $P b$ at a scale of $Q^{2}=100 \mathrm{GeV}^{2}$ relevant for pion production at transverse momentum $p_{T} \simeq 10 \mathrm{GeV}$.

The left-hand side of Fig. 11 shows the nuclear modification factors $R_{i}^{P b}$ for $u$ valence quarks, $\bar{u}$ sea quarks, and the gluon $g$ for two standard NLO sets of nPDFs: nDS (nuclear PDFs by De Florian-Sassot) [22] (solid lines) and EPS09 (nuclear PDFs by Eskola-Paukkunen-Salgado) [24] (dashed lines). The deviations of $R_{i}^{P b}$ from unity depend on the $x$ region and are typically referred to as "shadowing" $(x \lesssim 0.01)$, "antishadowing" $(0.01 \lesssim x \lesssim 0.2)$, "EMC effect" $(0.2 \lesssim x \lesssim 0.7)$, and "Fermi motion" $(x \gtrsim 0.7)$ in the terminology of nuclear deep inelastic scattering. Differences between the EPS09 and nDS sets are most pronounced for the gluon $\mathrm{nPDF}$, which plays a major role in $p P b$ collisions. Contrary to PDFs, the behavior of $\mathrm{nPDFs}$ is basically unconstrained below $x \simeq 0.01$ by present data, an $x$ region particularly prone to possible novel, nonlinear features of QCD scale evolution.

The corresponding medium modifications for FFs are displayed in the right panel of Fig. 11 and are distinctly different for quarks and gluons, where one finds suppression and enhancement, respectively, compared to vacuum fragmentation functions. The pattern is readily explained by the dominant role of quark fragmentation in describing the observed hadron attenuation in SIDIS off a heavy nucleus, while the enhancement of hadrons in $d A u$ collisions is closely linked with the gluon nFF [25]. In general, the observed nuclear effects are more pronounced for $\mathrm{nFFs}$ than for nPDFs, and nFFs can either enhance or overturn medium modifications computed with nPDFs but vacuum FF as was done, e.g., in Ref. [36].
Assuming that factorization holds in $p P b$ collisions at a c.m.s. energy of $\sqrt{S}=8.8 \mathrm{TeV}$, we present predictions for the production of neutral pions in Fig. 12 at NLO accuracy using nPDFs and the recently proposed set of nFFs [25] in Eq. (1). By construction, the entire nuclear dependence resides in the nonperturbative nPDFs and nFFs, and both the scale evolution and the partonic hard scattering cross sections $d \hat{\sigma}_{a b \rightarrow c X}$ are taken to be the same as in $p p$ collisions.

The upper left panel shows the $p_{T}$ dependence at central pseudorapidities $|\eta| \leq 0.5$ using $\mu_{f}=\mu_{f^{\prime}}=\mu_{r}=p_{T}$ in Eq. (1). We refrain from showing alternative results for different choices of $\mu_{f, f^{\prime}, r}$ as the theoretical scale ambiguities are very similar to the ones for $p p$ collisions discussed in Sec. III. Because of the steep fall of the cross section with $p_{T}$ over several orders of magnitude any differences between the NLO calculations based on various combinations of nPDFs and standard vacuum or medium modified FFs are hard to notice. The different patterns of nuclear modification become clearly visible, however, when expressed as ratios to the corresponding cross section in $p p$ collisions at the same c.m.s. energy, which are given in the lower left panel of Fig. 12.

The impact of medium induced effects on the hadronization process is clearly visible at larger $p_{T}$ by comparing the results obtained with the nDS set of nPDFs [22] along with nFFs [25] (solid line) and with DSS vacuum FFs [7]

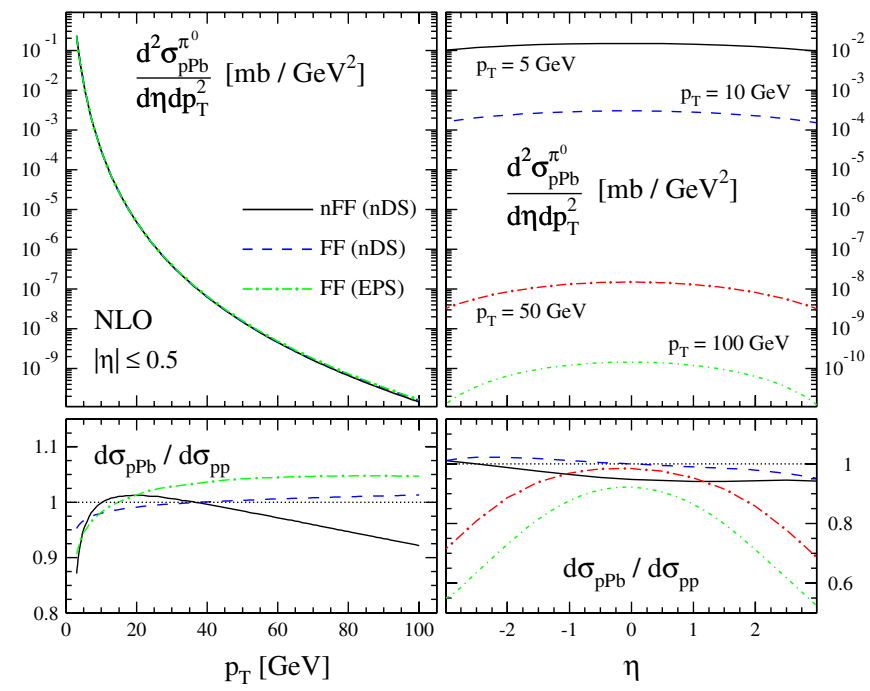

FIG. 12 (color online). Transverse momentum (left panels) and pseudorapidity (right panels) dependence of neutral pion production in $p P b$ collisions at $\sqrt{S}=8.8 \mathrm{TeV}$. The upper panels show the cross sections at NLO accuracy, and lower panels display the ratio to the corresponding cross section in $p p$ collisions. The $p_{T}$ dependent yields are integrated in $|\eta| \leq 0.5$ and computed for different combinations of nPDFs [22,24], nFFs [25], and vacuum FFs [7]. The $\eta$ differential results are given for various fixed values of $p_{T}$, and were obtained using the nDS set of nPDFs [22] and nFFs of [25]. 
(dashed line), leading to hadron attenuation and enhancement, respectively. The uncertainties in our present knowledge of nPDFs can be inferred from comparing the dot-dashed curve, which uses again the DSS vacuum FFs but now the EPS09 set of nPDFs [24], with the dashed line. The EPS nPDFs result in a somewhat more sizable enhancement than the nDS set due to the larger $R_{g}^{P b}$ at medium $x$ values, see Fig. 11, which are predominantly probed here; cf. Fig. 14 below. Notice that the EPS09 analysis [24] includes $d A u \rightarrow \pi^{0} X$ data from RHIC by assuming, however, that the entire nuclear dependence resides only in the initial state, i.e., in the nPDFs. This is clearly inadequate in view of the sizable hadron attenuation observed in semi-inclusive lepton-nucleus scattering [20]. Hence, in what follows we will only use the NLO nDS set of nPDFs, which was also obtained in a convolutional approach, very similar to the nFF analysis [25] based on Eq. (3).

The panels on the right-hand side of Fig. 12 show the pseudorapidity dependence of the $\pi^{0}$ yields at NLO accuracy for various fixed values of $p_{T}$. All results are obtained with the nDS set of nPDFs and nFFs of [25]. As before, we choose $\mu_{f}=\mu_{f^{\prime}}=\mu_{r}=p_{T}$ in Eq. (1). The upper panel displays the differential cross sections, and the lower one the ratios to the corresponding results in $p p$ collisions. The rapidity dependence of the $p P b$ nuclear modification factor is of great phenomenological relevance as it probes different ranges of momentum fraction $x_{b}$ in the nPDFs while the average value of $z$ in the fragmentation process only slowly increases with larger $|\eta|$; cf. Fig. 7. In addition, larger values of $p_{T}$ at fixed $\eta$ probe both on average larger $x_{a, b}$ and $z$ values. Pions produced in the backward $(\eta<0)$ direction of the proton beam require large momentum fractions $x_{b}$, and nPDFs are mainly probed in the antishadowing and EMC region. In contrast, forward hadrons are sensitive to smaller values of $x_{b}$ where shadowing is expected; see Fig. 11. In general, this leads to nuclear modification factors which are asymmetric in rapidity for any given value of $p_{T}$.

Based on these kinematic considerations, it was argued in Ref. [36] that a detailed study of the $p_{T}$ and $\eta$ dependent hadron yields at the LHC will help to determine nPDFs more precisely. However, the neglected medium modifications on the FFs can change the theoretical expectations for the ratio $d \sigma_{p P b} / d \sigma_{p p}$ significantly, as can be seen in Fig. 12. In particular, at larger $p_{T}$ and rapidities our expectations based on consistently including also nFFs in Eq. (1) show much larger nuclear modification factors than the few percent effects estimated in Fig. 2 of [36]. This is readily explained by the strong depletion of the quark FFs at large values of $z$ as displayed in Fig. 11, which is most relevant at large $\eta$ and $p_{T}$.

To elucidate this further, Fig. 13 gives more details on the relevance of different partonic subprocesses (a), the role of quarks and gluons in the hadronization (b), and the

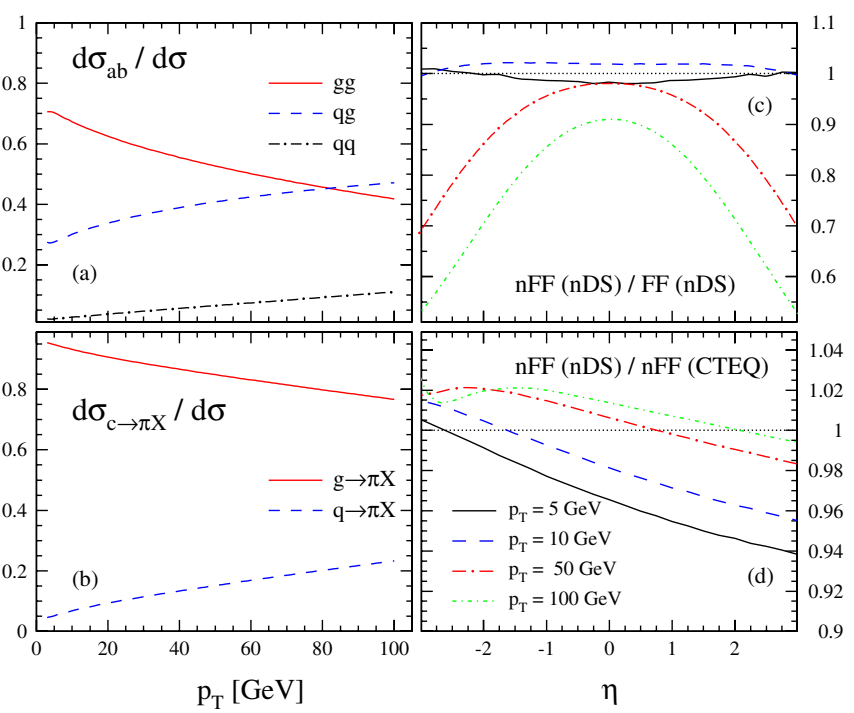

FIG. 13 (color online). (a): relative contributions of NLO partonic subprocesses $d \sigma_{a b}$ initiated by gluon-gluon, quarkgluon, and quark-quark scattering to the $p P b$ cross section shown in Fig. 12. (b): same as in (a) but now for the relative fractions of quarks and gluons fragmenting into the observed pion. (c) and (d) impact of medium modified FFs and nPDFs, respectively, on the rapidity dependence of the nuclear modification factors for different values of $p_{T}$ shown in the lower right panel of Fig. 12.

impact of nFFs (c) and nPDFs (d) on the rapidity dependence of the nuclear modification factor $d \sigma_{p P b} / d \sigma_{p p}$.

Panel (a) illustrates that in the entire $p_{T}$ range shown in Fig. 12 gluon initiated subprocesses dominate the cross section at central rapidities. Most of the pions are produced from gluon fragmentation as can been seen in Fig. 13(b). Only for $p_{T} \gtrsim 50 \mathrm{GeV}$ quark nFFs contribute at the level of $20 \%$ or more. For hadrons produced at larger $\eta$, the fractions in (a) and (b) shift somewhat in favor of quarkgluon scattering and quark fragmentation, respectively.

Figures 13(c) and 13(d) make explicit that the dominant medium effect on $d \sigma_{p P b} / d \sigma_{p p}$ resides indeed in the finalstate. Panel (c) shows the ratio where the denominator is computed with the nDS set of nPDFs such that the entire deviation from unity is due to the nFFs. Likewise, in panel (d) we compute $d \sigma_{p p}$ in the nuclear modification factor with nFFs rather than vacuum FFs. This quantifies medium effects due to the initial-state nPDFs. The latter effect is much smaller, and our results agree with the LO estimates shown in Ref. [36]. Thus we believe that singleinclusive hadron production at the LHC will provide a decisive test of the proposed factorized framework for $p P b$ collisions and the concept of medium modified FFs. In particular, the ALICE experiment is capable of identifying different hadron species in a wide range of rapidity which will greatly facilitate such theoretical studies. To disentangle and characterize nuclear effects in PDFs and in FFs precisely, requires to study also other hard processes in 
$p P b$ collisions not affected by hadronization like jet production, Drell-Yan, or prompt photons. Such measurements will provide an important input to future global QCD analyses of nPDFs.

Finally, Fig. 14 gives an idea which values of $x_{a, b}$ and $z$ are probed on average in the $p_{T}$ differential cross section at central rapidities shown in Fig. 12. As for $p p$ collisions, the estimates are based on Eq. (4). It turns out that measurements of pion production up to transverse momenta of $100 \mathrm{GeV}$ probe both the nPDFs and the usual PDFs in the range of momentum fractions from 0.01 to 0.1 . To access smaller values in the lead nucleus, where novel, nonlinear features of QCD scale evolution may become relevant, one needs to go to very forward pseudorapidities [32]. From the lower panel of Fig. 14 one can infer that $p P b$ collisions are mainly sensitive to fairly large values of momentum fraction taken by the produced pion, with $\langle z\rangle$ slightly increasing with $p_{T}$. This is similar to what was observed in $d A u$ collisions at RHIC energies [25] and for $p p$ collisions above in Fig. 7. Despite the large c.m.s. energy of $\sqrt{S}=8.8 \mathrm{TeV}$, (n)FFs can be safely applied as one is fairly insensitive to the region of small $z$, where the concept of (n) FFs is bound to fail due to finite hadron mass effects, higher twist contributions, and the singular behavior of the timelike

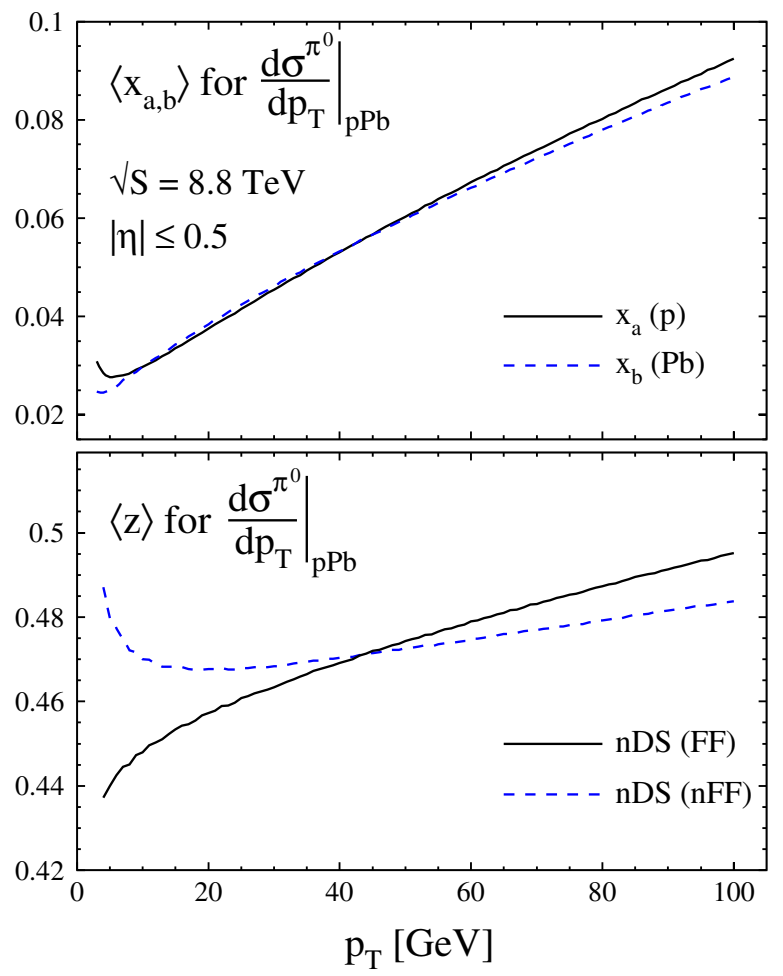

FIG. 14 (color online). Upper panel: mean values of $x_{a}$ and $x_{b}$ probed in the proton and the lead nucleus, respectively, for pion production at central rapidities in $p P b$ collisions at $\sqrt{S}=$ 8.8 TeV. Lower panel: same as above but now for the mean value of $z$ computed with the nDS set of nuclear PDFs and DSS vacuum FFs [7] (solid line) and nFFS of Ref. [25] (dashed line). evolution kernels. We note that one samples slightly different values $\langle z\rangle$ in the nuclear and vacuum FFs as can been seen by comparing the dashed and solid lines.

\section{SUMMARY AND CONCLUSIONS}

We have presented a comprehensive analysis of singleinclusive hadron production in $p p$ and $p P b$ collisions at LHC energies based on QCD factorization.

It was shown that first results from the LHC experiments for charged hadron spectra agree well with expectations based on NLO pQCD calculations using latest sets of parton distribution and fragmentation functions.

Based on this success, we have given detailed predictions for various kinematic distributions of identified and unidentified hadrons in $p p$ collisions. Different sources of theoretical uncertainties were discussed and estimated. It turned out that the residual factorization and renormalization dependence of the cross sections at NLO accuracy represents the dominant source of uncertainty, in particular, at small values of transverse momentum of the produced hadron. Approximate $x_{T}$ scaling with $n \simeq 5$ can be accommodated within the rather large theoretical scale ambiguity. Uncertainties from fragmentation functions for different hadron species were propagated to the single-inclusive yields based on the robust Lagrange multiplier method and found to be sizable, although smaller than those associated with the truncation of the perturbative series at NLO.

To elucidate the possible impact of upcoming hadron production data from the LHC on future global analyses of fragmentation functions, we have studied in detail the relative contributions of different partonic subprocesses and the fractions of quark and gluon fragmentation into the observed hadrons as functions of transverse momentum and pseudorapidity. In addition, we have estimated the mean values of momentum fractions both in the parton densities functions and in the fragmentation process which are relevant at LHC energies. It was found that like at hadron colliders at lower energies, most of the produced hadrons take a rather large fraction of the parent parton's momentum which ensures the applicability of the concept of factorized fragmentation functions also at LHC energies.

Finally, we have proposed a set of measurements of single-inclusive hadron production in proton-lead collisions to shed light on the so far poorly understood hadronization mechanism in a nuclear medium. We have presented expectations for pion yields at NLO accuracy based on standard QCD factorization using sets of medium modified parton distribution and fragmentation functions.

Single-inclusive hadron production in an unprecedented energy range at the LHC will challenge our current understanding of fragmentation functions and help to further constrain them, in particular, at large momentum fractions. The large range of transverse momenta of the produced hadrons will allow for detailed studies of the scale evolution 
for fragmentation functions. Data obtained in proton-lead collisions will explore the limits of characterizing nuclear modifications of hadron production yields in a factorized QCD approach and scrutinize the applicability of the recently proposed concept of medium modified fragmentation functions.

\section{ACKNOWLEDGMENTS}

We thank Z. Trocsanyi and W. Bell for their help with the CMS and ATLAS data. This work was partially supported by CONICET, ANPCyT, UBACyT, BMBF, and the Helmholtz Foundation.
[1] See, e.g., J.C. Collins, D. E. Soper, and G. Sterman, Perturbative $Q C D$, edited by A.H. Mueller, Adv. Ser. Direct. High Energy Phys., Vol. 5, (World Scientific, Singapore, 1989) p. 1, and references therein.

[2] P. M. Nadolsky et al., Phys. Rev. D 78, 013004 (2008).

[3] A. D. Martin, W. J. Stirling, R. S. Thorne, and G. Watt, Eur. Phys. J. C 63, 189 (2009).

[4] R. D. Ball et al., Nucl. Phys. B838, 136 (2010).

[5] P. M. Nadolsky et al., Phys. Rev. D 78, 013004 (2008).

[6] D. Stump et al., Phys. Rev. D 65, 014012 (2001).

[7] D. de Florian, R. Sassot, and M. Stratmann, Phys. Rev. D 75, 114010 (2007).

[8] D. de Florian, R. Sassot, and M. Stratmann, Phys. Rev. D 76, 074033 (2007).

[9] S. Albino, B. A. Kniehl, and G. Kramer, Nucl. Phys. B803, 42 (2008).

[10] D. de Florian, R. Sassot, M. Stratmann, and W. Vogelsang, Phys. Rev. Lett. 101, 072001 (2008); Phys. Rev. D 80, 034030 (2009).

[11] See, e.g., F. Arleo, Eur. Phys. J. C 61, 603 (2009); A. Accardi, F. Arleo, W. K. Brooks, D. D'Enterria, and V. Muccifora, Riv. Nuovo Cimento Soc. Ital. Fis. 032, 439 (2010).

[12] S. J. Brodsky and G. R. Farrar, Phys. Rev. Lett. 31, 1153 (1973); D. W. Sivers, S. J. Brodsky, and R. Blankenbecler, Phys. Rep. 23, 1 (1976); R. Blankenbecler, S. J. Brodsky, and J. F. Gunion, Phys. Rev. D 18, 900 (1978).

[13] F. Arleo, S. J. Brodsky, D. S. Hwang, and A. M. Sickles, Phys. Rev. Lett. 105, 062002 (2010).

[14] J.C. Collins and D.E. Soper, Nucl. Phys. B193, 381 (1981); B213, 545(E) (1983); B194, 445 (1982).

[15] M. Stratmann and W. Vogelsang, Phys. Rev. D 64, 114007 (2001).

[16] G. Curci, W. Furmanski, and R. Petronzio, Nucl. Phys. B175, 27 (1980); W. Furmanski and R. Petronzio, Phys. Lett. 97B, 437 (1980); L. Beaulieu, E. G. Floratos, and C. Kounnas, Nucl. Phys. B166, 321 (1980); M. Stratmann and W. Vogelsang, Nucl. Phys. B496, 41 (1997); S. Moch and A. Vogt, Phys. Lett. B 659, 290 (2008).

[17] F. Aversa, P. Chiappetta, M. Greco, and J. P. Guillet, Nucl. Phys. B327, 105 (1989); D. de Florian, Phys. Rev. D 67, 054004 (2003); B. Jäger, A. Schäfer, M. Stratmann, and W. Vogelsang, Phys. Rev. D 67, 054005 (2003).

[18] T. Aaltonen et al. (CDF Collaboration), Phys. Rev. D 79, 112005 (2009).
[19] S. Albino, B. A. Kniehl, and G. Kramer, Phys. Rev. Lett. 104, 242001 (2010); F. Arleo, D. d'Enterria, and A. S. Yoon, J. High Energy Phys. 06 (2010) 35; M. Cacciari, G. P. Salam, and M. J. Strassler, arXiv:1003.3433.

[20] A. Airapetian et al. (HERMES Collaboration), Nucl. Phys. B780, 1 (2007); Phys. Lett. B 684, 114 (2010).

[21] J. Adams et al. (STAR Collaboration), Phys. Lett. B 616, 8 (2005); 637, 161 (2006); S. S. Adler et al. (PHENIX Collaboration), Phys. Rev. Lett. 98, 172302 (2007); B. I. Abelev et al. (STAR Collaboration), Phys. Rev. C 81, 064904 (2010).

[22] D. de Florian and R. Sassot, Phys. Rev. D 69, 074028 (2004).

[23] M. Hirai, S. Kumano, and T. H. Nagai, Phys. Rev. C 76, 065207 (2007).

[24] K. J. Eskola, H. Paukkunen, and C. A. Salgado, J. High Energy Phys. 04 (2009) 065.

[25] R. Sassot, M. Stratmann, and P. Zurita, Phys. Rev. D 81, 054001 (2010).

[26] X. F. Guo and X. N. Wang, Phys. Rev. Lett. 85, 3591 (2000); N. Borghini and U.A. Wiedemann, arXiv:hepph/0506218; A. Majumder, E. Wang, and X.N. Wang, Phys. Rev. C 73, 044901 (2006); N. Armesto, L. Cunqueiro, C. A. Salgado, and W. C. Xiang, J. High Energy Phys. 02 (2008) 048; S. Domdey et al., Nucl. Phys. A808, 178 (2008); S. Albino, B. A. Kniehl, and R. Perez-Ramos, Nucl. Phys. B819, 306 (2009).

[27] A. Accardi et al., arXiv:hep-ph/0308248.

[28] G. Aad et al. (ATLAS Collaboration), Phys. Lett. B 688, 21 (2010).

[29] V. Khachatryan et al. (CMS Collaboration), J. High Energy Phys. 02 (2010) 41; Phys. Rev. Lett. 105, 022002 (2010).

[30] K. Aamodt et al. (ALICE Collaboration), arXiv:1007.0719.

[31] K. Aamodt et al. (ALICE Collaboration), Eur. Phys. J. C 65, 111 (2010); 68, 89 (2010); 68, 345 (2010).

[32] V. Guzey, M. Strikman, and W. Vogelsang, Phys. Lett. B 603, 173 (2004).

[33] See, e.g., S. J. Brodsky, G. P. Lepage, and P. B. Mackenzie, Phys. Rev. D 28, 228 (1983).

[34] A. Adare et al. (PHENIX Collaboration), Phys. Rev. D 76, 051106 (2007); 79, 012003 (2009); A. Bazilevsky, "APS Spring Meeting 2010."

[35] See, e.g., D. de Florian and W. Vogelsang, Phys. Rev. D 71, 114004 (2005).

[36] P. Quiroga-Arias, J. G. Milhano, and U. A. Wiedemann, Phys. Rev. C 82, 034903 (2010). 